\title{
Software compensation for lung volume in assessment of inspiratory muscle strength and endurance
}

\author{
D K McKenzie, S C Gandevia, R B Gorman, J B Leeper
}

\begin{abstract}
Background - To increase the sensitivity of measurements of maximal inspiratory pressure (MIP) as a test of inspiratory muscle strength and endurance, software was developed to correct for variation in lung volume.

Methods - Using a body plethysmograph to determine absolute lung volume during each manoeuvre, values for MIP were expressed as a percentage of the pressure "expected" from the unfatigued MIP/lung volume relation in each subject.

Results - The method reduced the variance in peak and average pressure during a series of 18 MIP manoeuvres of 10 seconds duration separated by rest intervals of 10 seconds.

Conclusions - The correlation between average pressure and contraction number was improved significantly by the MIP/ lung volume correction. This simple correction has many applications in measurements of the MIP.

(Thorax 1995;50:230-234)
\end{abstract}

Keywords: maximal inspiratory pressure, diaphragm, lung volume, muscle strength.

The force developed in an isometric maximal voluntary contraction may be used to assess muscle strength while the decline in maximal voluntary contraction is a measure of fatigue. Inspiratory muscle is traditionally assessed by maximal quasi-isometric inspiratory (Mueller) efforts against an occluded airway at or near residual volume, ${ }^{12}$ but the maximal inspiratory pressure (MIP) depends on initial lung volume. $^{3-6}$ Variable voluntary drive to the inspiratory muscles may also contribute to variability in the MIP. ${ }^{7-9}$

Muscle fatigue is defined as any decline in force (or velocity) generating capacity regardless of the force required for the task in hand. ${ }^{1011}$ If inspiratory muscle fatigue is assessed by the decline in MIP, however, the result will be affected by changes in lung volume as well as fatigue. Variations in initial lung volume during a test of endurance may be reduced using feedback of the chest wall shape and/or oesophageal pressures, ${ }^{12}$ but in some studies lung volume is varied as part of the experimental protocol. ${ }^{1213}$

We describe a simple computerised method in which the relation between the unfatigued MIP and thoracic gas volume (TGV) is used to normalise the maximal pressures achieved in a test of inspiratory muscle endurance. A graphical method had formerly been used to examine the influence of lung volume on inspiratory muscle endurance ${ }^{13}$ and to compare respiratory muscle endurance in patients with chronic airflow limitation and control subjects. ${ }^{14}$ However, that method was slow and relatively inaccurate. The present method was used in a study of inspiratory muscle endurance in asthmatic subjects in whom lung volume varied both voluntarily and with histamine induced bronchoconstriction. ${ }^{15}$ Details of this method are outlined below together with an evaluation of its effectiveness in six healthy subjects.

\section{Methods}

The automated correction method described uses the relation between MIP and TGV which is determined for each subject using a pressure compensated, integrated flow body plethysmograph. TGV is derived during single inspiratory efforts against a closed pneumatic shutter. ${ }^{16}$ Flow through the plethysmograph opening is measured with a pneumotachograph; integrated flow (volume) is calibrated with a three litre syringe for standard measurement and with a $100 \mathrm{ml}$ syringe for the relatively small volume changes when the shutter is closed. When the shutter closes the volume signal is automatically zeroed and amplified tenfold to improve resolution. Mouth pressure is measured with a differential pressure transducer and calibrated with a $100 \mathrm{~cm} \mathrm{H}_{2} \mathrm{O}$ manometer. The MIP and the initial TGV during each occluded inspiratory effort are determined by a microcomputer sampling at $100 \mathrm{~Hz}$ by applying Boyle's law to the mouth pressure and volume signals using programs written in the ASYST programming language (Keithley Instruments Inc, USA). The flow chart is given in fig $1 \mathrm{~A}$.

The control (unfatigued) MIP/TGV relation is obtained from a series of maximal inspiratory contractions (2-3 seconds duration) after exhalation from near total lung capacity while the experimenter monitors mouth pressure and the relative change in lung volume on an $X-Y$ oscilloscope. The experimenter can observe the subject's technique of pressure development and decide whether the trial was technically acceptable. For example, closure of the epiglottis associated with buccal suction ("cheek artefact") is detected readily as a large pressure change with an inappropriately small volume change - that is, no lung decompression. Leaks around the mouthpiece are also detected as an 
A

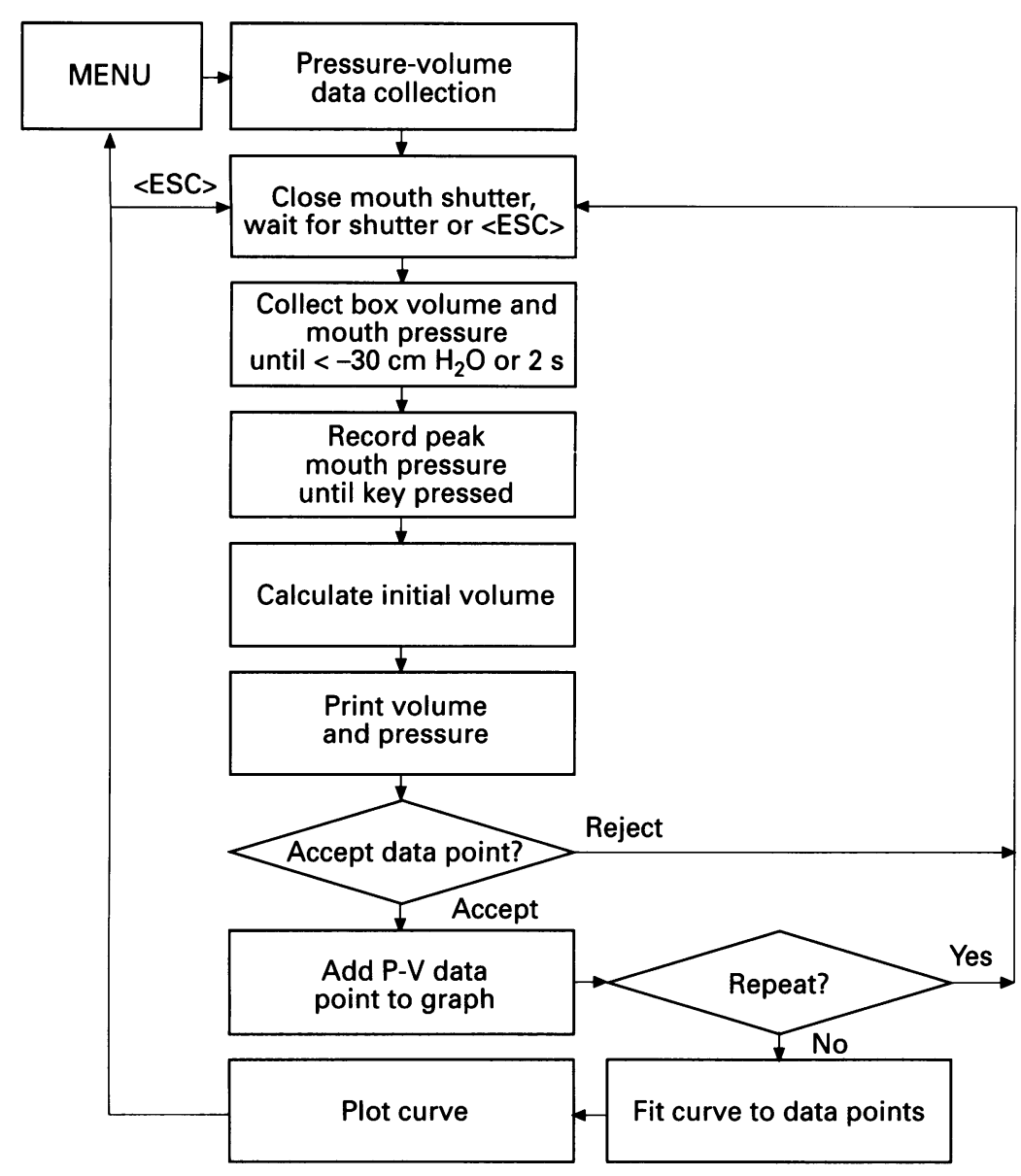

B

$\Delta$ Volume (litres)

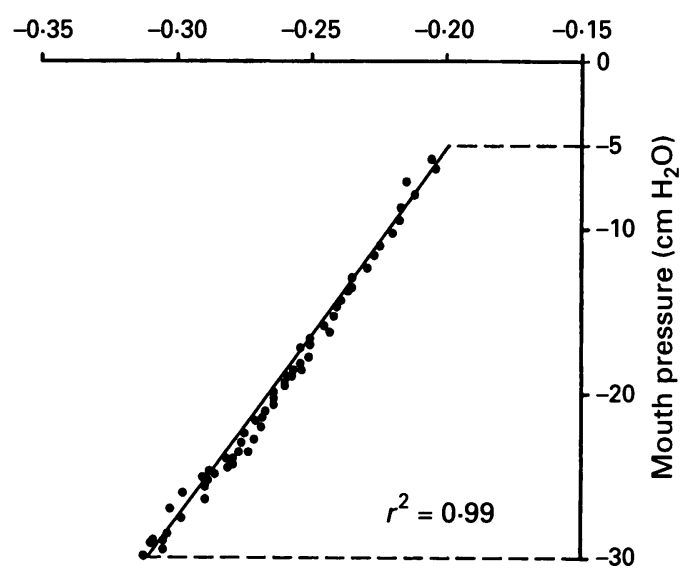

Figure 1 (A) Flow chart for the computer program that calculates thoracic gas volume from the pressure-volume relation and Boyle's law during MIP manoeuvres.

(B) Relationship between mouth pressure and change in box volume during a typical MIP manoeuvre with data sampled by computer at $100 \mathrm{~Hz}$.

inappropriate volume change associated with non-linearity of the pressure-volume trace. The TGV at closure of the shutter is calculated in the initial phase of each occluded effort $(-5$ to $-30 \mathrm{~cm} \mathrm{H}_{2} \mathrm{O}$; fig 1B). This range was chosen to avoid pressure artefacts immediately after closure of the pneumatic shutter and possible non-linearity of the volume-pressure relation at high negative pressures. The number of data points collected in each trial depends on the rate of inspiratory pressure development; usually 9-15 points are obtained for pressures between -5 and $-30 \mathrm{~cm} \mathrm{H}_{2} \mathrm{O}$. If the MIP does not reach $-30 \mathrm{~cm} \mathrm{H}_{2} \mathrm{O}$ the data points collected during the first two seconds of the contraction are used to fit the regression. A linear regression is fitted to the points and the slope obtained. The correlation coefficient is usually greater than 0.980 (fig 1B). Initial $\mathrm{TGV}$ is then calculated by applying Boyle's law with the slope and initial pressure. Final lung volume at the peak inspiratory pressure is then derived by a Boyle's law correction of the initial volume. After each contraction MIP is displayed graphically as a function of final TGV (at peak pressure) using a different symbol to distinguish the most recent data point. The MIP and TGV coordinates are also printed on the screen.

The menu includes a "set-up" option, which can be called up at any time, that allows variation of the pressure range used to determine TGV for different subjects. The set-up option also allows alteration of the axes for the plot of MIP versus TGV to cater for a wide range of volumes and pressures. Efforts which are obviously submaximal, in comparison with others at a similar lung volume, or contaminated by "cheek artefact" (glottic closure) can be deleted on-line or, subsequently, with a cursordriven editing program. The MIP/TGV relation is then approximated from 10-15 acceptable efforts with a second order polynomial using a least squares method (fig 2).

\section{APPLICATION TO ENDURANCE TESTS}

The MIP/TGV relation is used to apply volume correction to values of MIP obtained during endurance tests. Six subjects (four men) aged 21-41 participated in the study undertaken to assess the efficacy of the method. Apart from two of the authors, subjects were unfamiliar with the required manoeuvres. The protocol described consisted of 18 sustained maximal Mueller manoeuvres, but the method can be applied to any test which includes measurement of MIP. Each contraction lasted 10 seconds with rest intervals of 10 seconds. Subjects were continually encouraged to maintain maximal

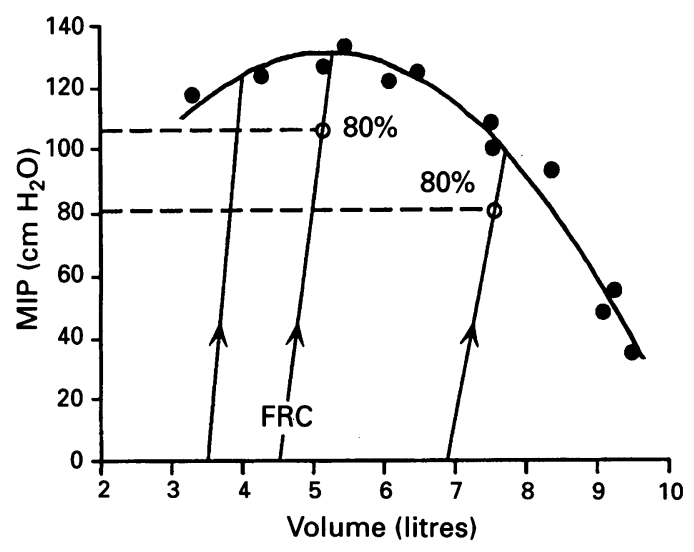

Figure 2 Control relation between absolute lung volume and maximal inspiratory pressure (MIP) for one subject. Data fitted by a second order polynomial $(r=0.985)$. Solid lines indicate the volume-pressure profile during efforts at FRC (middle line) and at the extreme initial volumes from the endurance test. The dashed lines illustrate the potential error, without correction, for efforts performed with the muscles fatigued such that they can produce only $80 \%$ of maximal pressure. 

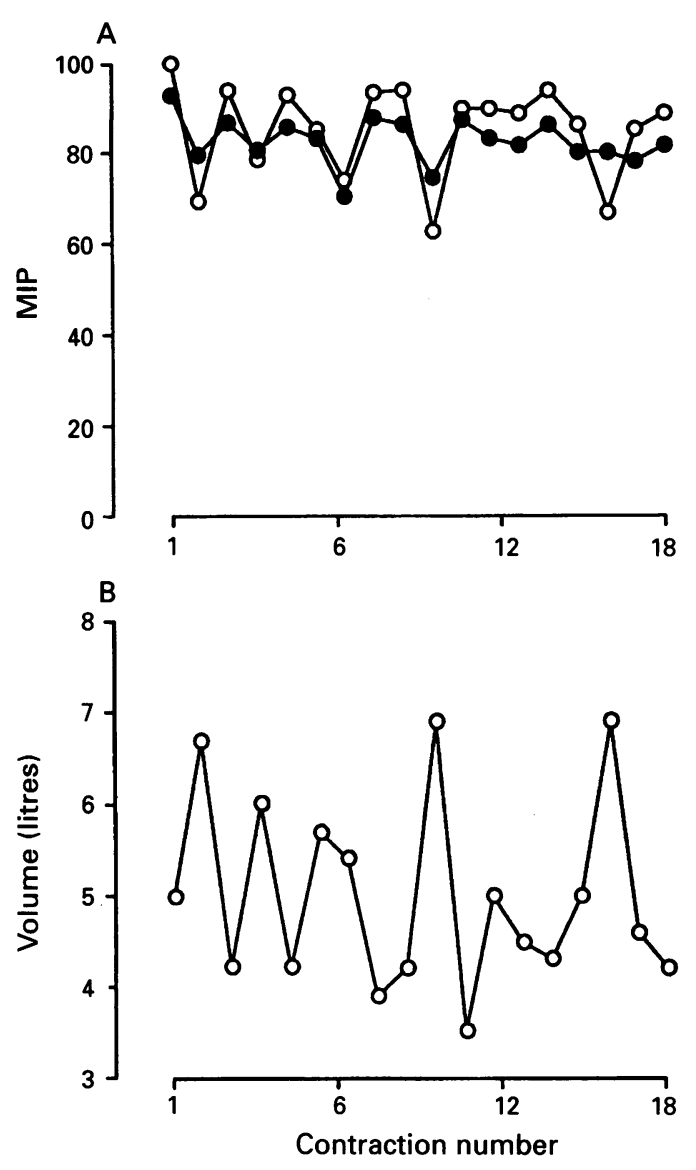

Figure 3 An endurance test consisting of 18 maximum inspiratory pressure (MIP) manoeuvres of 10 seconds duration, $50 \%$ duty cycle (same subject as in fig 2). (A) MIP normalised to the best of the first three contractions (O, "\% max"). (B) Initial lung volume for each MIP. = values for MIP expressed as "\% expected" at that lung volume using the control MIP/volume relation (fig 2).

effort throughout each contraction and received visual feedback of mouth pressure. Breathing pattern was unconstrained between contractions.

For each contraction MIP and mouth pressure averaged over 10 seconds were measured and the initial and final TGV were calculated (as described above). The MIP expected at that final lung volume was then derived from the curve describing the control (unfatigued) MIP/TGV relation. Maximal pressures were expressed as percentages of the expected MIP values. Any value below $100 \%$ would therefore indicate fatigue and/or a reduced level of voluntary activation.

Effect of lung volume correction on the peak and average maximum inspiratory pressure (MIP) measured during 18 contractions

\begin{tabular}{lccccc}
\hline & \multicolumn{2}{l}{ Peak MIP } & & \multicolumn{2}{c}{ Average MIP } \\
\cline { 2 - 3 } \cline { 5 - 6 } & Uncorrected & Corrected & & Uncorrected & Corrected \\
\hline Mean SD $^{1}$ & 17.0 & 11.9 & 18.1 & 13.8 \\
Sum sq. diff. $^{2}$ & 539 & 082 & & 473 & 146 \\
Exponential fit $(r)^{3}$ & 0.466 & 0.488 & & 0.784 & 0.908 \\
Probability $^{4}$ & $<0.10$ & $<0.05$ & & $<0.01$ & $<0.001$ \\
\hline
\end{tabular}

${ }^{1}$ Grand mean of the SDs for relevant data in fig 3.

${ }^{2}$ Sum of the squares of the differences based on data in fig 3 and an exponential fit.

${ }^{3}$ Correlation coefficient for the fitted exponential.

${ }^{4}$ Statistical probability for the exponential fit.

\section{Results}

The range of pressures used for fitting the relation between absolute lung volume and MIP in six subjects was $38-155 \mathrm{~cm} \mathrm{H}_{2} \mathrm{O}$. The polynomial equation provided a good fit to the TGV/MIP relation in all subjects - for example, fig 2; correlation coefficients 0.98-0.99.

Figure 3 shows an example of an endurance test in which the variation in lung volume was deliberately exaggerated during a series of 18 maximal inspiratory efforts each of 10 seconds duration. Initial lung volume varied by about 3.41 (TGV 3.5-6.91). This could account for a variation in MIP of $25 \mathrm{~cm} \mathrm{H}_{2} \mathrm{O}$ (fig 2, approximately $20 \%$ of maximal voluntary contraction), greater than the decline which might occur due to fatigue. ${ }^{13}$ When the pressure was corrected for lung volume the variability in MIP was reduced but not entirely eliminated.

Group data for the endurance test are shown in fig 4. Values for peak pressure (MIP) and average pressure over each 10 second contraction are shown with and without the correction for variation in lung volume. The lung volume varied from $25 \%$ to $97 \%$ of the vital capacity for the group of six subjects. A simple exponential fit applied to each data set - for example, fig $4 \mathrm{~A}$ - was used to assess the reduction in MIP with successive contractions. Correction for variation in initial lung volume reduced the variance of the data and improved the correlation coefficients (table).

\section{Discussion}

We describe a simple method to correct for the influence of variation in lung volume during repeated measurements of MIP. This method can be applied to any test of inspiratory muscle strength or endurance. The derived variable, termed the percentage of "expected" pressure, applies across a wide range of lung volumes. With this correction other factors which affect MIP can be assessed more sensitively.

The on-line method described here has the advantage of speed, displaying the absolute lung volume, absolute MIP, and the corrected value almost immediately. The only alternative method would be to measure the exhaled volume from total lung capacity. This is impossible in many protocols, however, either because of diaphragmatic fatigue or because of a requirement to sustain hyperpnoeic levels of ventilation. The use of plethsymographic volume signal also allows "cheek artefact" to be detected instantly. Although a cursor-driven editing program was used here to eliminate segments with glottic closure, this process can also be automated.

One limitation of the method is the need for a body plethysmograph. The subject is then less readily accessible for other types of intervention such as transcutaneous nerve stimulation, but in most instances this problem can be overcome. For example, we have performed bilateral phrenic nerve stimulation remotely via hookwire electrodes introduced with hypodermic needles and recorded transdiaphragmatic pressure, ${ }^{1718}$ and have induced bronchoconstriction in asthmatic subjects 
A
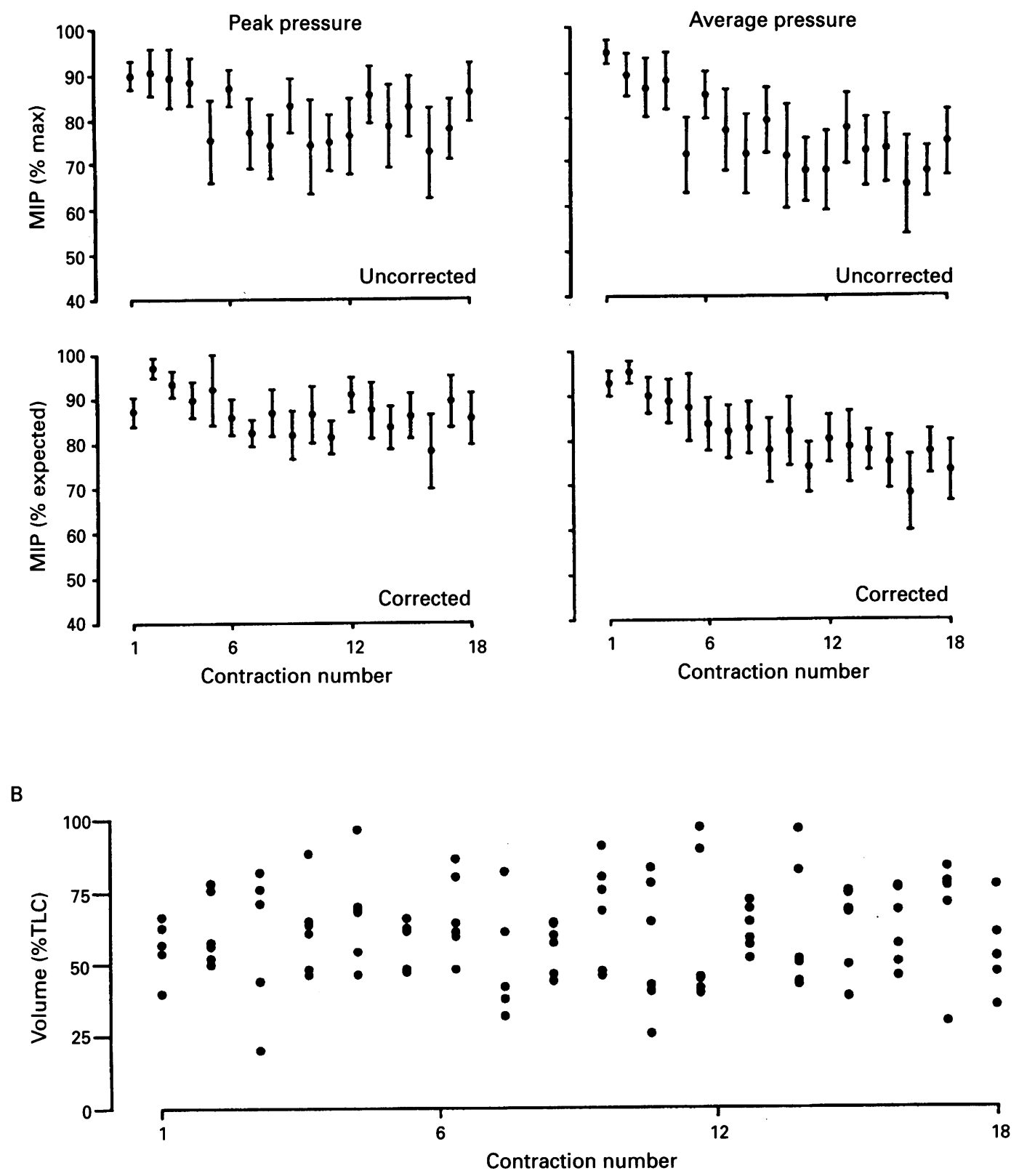

Figure $4(A)$ Mean (SD) group data for peak inspiratory pressure and average inspiratory pressure sustained during each of 18 MIP manoeuvres normalised to the best of the first three contractions for each subject $(n=6)$. Lower panels show the same data corrected for variation in lung volume by expressing values as percentage expected from the unfatigued MIP/volume relation. (B) Initial volume expressed as percentage of total lung capacity (TLC) for each subject for each contraction. Note that the wide scatter in lung volume was produced to assess the correction method.

seated in a plethysmograph using nebulised histamine. ${ }^{15}$ For prolonged endurance studies the door of the plethysmograph can be intermittently opened and the temperature can be controlled using an air conditioner. Temperature "drift" of the integrated volume signal is a minor problem for monitoring, but it can be overcome by adjusting a variable DC offset or a reset button on the integrator. Indeed, the main reason for developing the Boyle's law method described here was to enable an accurate measure of TGV independent of any real or artefactual change in the end expiratory level.

The reliability of the lung volume correction depends on the accuracy of fit of the polynomial to the MIP/TGV data points. Subsequent tests must be performed within the volume range of data points to avoid errors from extrapolation. The representative data in fig 3 shows that the correction appears to undercompensate for the reduced pressures obtained at very high lung volumes. This may reflect a systematic variation in voluntary drive with lung volume. The decrease in MIP values at volumes below functional residual capacity is also suggestive of incomplete voluntary activation. ${ }^{9}$ The data in the table suggest that the correction was more effective for the measurement of average pressure than for the peak values. This reflects the greater relative decline in average pressure over the series of 18 contractions compared with peak pressure so that the data were more appropriate for an exponential fit regardless of the scatter of data points. The improvement in scatter was similar for both data sets. 
This study was supported by the National Health and Medical Research Council of Australia and the Asthma Foundation of New South Wales. Copies of the software are available from our laboratory.

1 Black LF, Hyatt RE. Maximal respiratory pressures: normal values and relationship to age and sex. Am Rev Respir Dis 1969;99:696-702.

2 Rochester DF, Braun NMT, Arora, NS. Respiratory muscle strength in chronic obstructive pulmonary disease. $\mathrm{Am}$ Rev Respir Dis 1979;119(Suppl):151-4.

3 Rohrer, F. Der Zusammenhang der Atemkraefte und ihre Abhaengigkeit vom Dehnungszustand der Atmungsorgane. Pflügers Arch Ges Physiol 1916;165:419-44.

4 Rahn H, Otis AB, Chadwick LE, Fenn WO. The pressurevolume diagram of the thorax and lung. Am $\mathfrak{f}$ Physiol 1946;242: $\mathrm{H} 1-6$

5 Cook CD, Mead J, Orzalesi MM. Static volume-pressure characteristics of the respiratory system during maximal efforts. F Appl Physiol 1964;19:1016-22.

6 Agostoni E, Mead J. Statics of the respiratory system. In: Fenn WO, Rahn H, eds. Handbook of physiology, Section 3, Volume 1; Respiration. Washington: American Physiological Society, $1964 ; 404$

7 Gandevia SC, McKenzie DK. Activation of the human diaphragm during maximal static efforts. $f$ Physiol (Londiaphragm during maxim

8 Gandevia SC, McKenzie DK, Plassman BL. Activation of human respiratory muscles during different voluntary manoeuvres. F Physiol (London) 1990;428:387-403.
9 Hershenson MB, Kikuchi Y, Loring SH. Relative strengths of the chest wall muscles. $\mathcal{F}$ Appl Physiol 1988;65:852-62

10 Bigland-Ritchie B. Muscle fatigue and the influence of changing neural drive. Clin Chest Med 1984;5:21-34.

11 NHLBI Workshop. Respiratory muscle fatigue: report of the respiratory muscle fatigue workshop group. Am Rev the respiratory muscle fatig

12 Roussos C, Fixley M, Gross D, Macklem PT. Fatigue of inspiratory muscles and their synergic behaviour. $\mathscr{F} A p p l$ Physiol 1979;46:897-904.

13 McKenzie DK, Gandevia SC. Influence of muscle length on human inspiratory and limb muscle endurance. Respir Physiol 1987;67:171-82.

14 Newell SZ, McKenzie DK, Gandevia SC. Inspiratory and skeletal muscle strength and endurance and diaphragmatic activation in patients with chronic airflow limitation. Thorax 1989;44:903-12.

15 Gorman RB, McKenzie DK, Gandevia SC, Plassman BL. Inspiratory muscle strength and endurance during hyperinflation and histamine induced bronchoconstriction. Thorax 1992;47:922-7.

6 Desmond KJ, Demizio DL, Allen PD, Beaudry PH, Coates AL. An alternative method for the determination of functional residual capacity in a plethysmograph. Am Rev Respir Dis 1988;137:273-6.

17 Allen GM, McKenzie DK, Gandevia SC, Bass S. Reduced voluntary drive to breathe in asthmatic subjects. Respir Physiol 1993;93:29-40.

18 Hubmayr RD, Litchy WJ, Gay PC, Nelson SB. Transdiaphragmatic twitch pressure. Effects of lung volume and chest wall shape. Am Rev Respir Dis 1988;139:647-52. 